\title{
Subcarrier Wave Quantum Key Distribution in Telecommunication Network with Bitrate 800 kbit/s
}

\author{
A.V. Gleim ${ }^{a}$, Yu.V. Nazarov, V.I. Egorov, S.V. Smirnov, O.I. Bannik, V.V. Chistyakov, S.M. Kynev, A.A. \\ Anisimov, S.A. Kozlov, and V.N. Vasiliev \\ ITMO University, 49 Kronverksky pr., Saint Petersburg 197101, Russia
}

\begin{abstract}
In the course of work on creating the first quantum communication network in Russia we demonstrated quantum key distribution in metropolitan optical network infrastructure. A single-pass subcarrier wave quantum cryptography scheme was used in the experiments. BB84 protocol with strong reference was chosen for performing key distribution. The registered sifted key rate in an optical cable with $1.5 \mathrm{~dB}$ loss was $800 \mathrm{Kbit} / \mathrm{s}$. Signal visibility exceeded $98 \%$, and quantum bit error rate value was $1 \%$. The achieved result is a record for this type of systems.
\end{abstract}

Keywords: quantum cryptography, sideband, optical fiber, telecom network.

Quantum cryptography (QC) systems allow distribution of secure symmetric key between several parties by using single photons [1]. Recent success in developing experimental QC setups has been followed by a growing interest in integrating these systems into installed optical fiber telecommunication networks, which remains an important condition for wider use of the QC technology. Today pilot quantum network projects have been launched in Europe, the USA, Japan and China [2,3]. The record operating distance and bitrate achieved for QC in optical network cables are $83 \mathrm{~km}$ and $300 \mathrm{Kbit} / \mathrm{s}$ (line loss $14.5 \mathrm{~dB}$ ) [3], respectively.

Earlier we demonstrated a subcarrier wave QC system that allows performing key distribution at $180 \mathrm{bit} / \mathrm{s}$ rate in an optical fiber channel with $35 \mathrm{~dB}$ loss using two-state phase protocol [4]. Advantages of this type of systems [5] include one-pass setup (simplifying network commutation), lack of interferometric schemes in the receiver module (high stability and robustness) and possibility of transmitting up to 10 independent quantum channels in a single DWDM multiplexer window, thus increasing the spectral efficiency of the secure channel by an order of magnitude [6]. It makes subcarrier wave QC systems promising as quantum communication network nodes.

The scope of current work was demonstrating subcarrier wave QC system operation in an installed metropolitan optical network. In order to perform this task, the system [4] was significantly improved: we implemented a four-state phase protocol, added optical synchronization, and introduced a singlepass scheme for compensating polarization distortion of the optical signal travelling thought a communication cable.

Based on the reworked setup, we implemented two metropolitan quantum network nodes situated in Saint Petersburg in ITMO University buildings at Kadetskaya line $3 \mathrm{~b}$ and Birzhevaya line 14 (Fig.

${ }^{\text {a }}$ Corresponding author: aglejm@yandex.ru 
1). Conventional optical lines and telecom equipment were used in the experiment. Key distribution was performed in single-channel mode through a standard optical cable with total loss $1.5 \mathrm{~dB}$ corresponding to $1 \mathrm{~km}$ line length. Experimentally achieved key generation rate was $800 \mathrm{Kbit} / \mathrm{s}$, with mean quantum bit error rate value not exceeding $1 \%$. We observed stable operation of the setup for 1 hour. These preliminary results suggest that the developed system can exchange keys in fiber optical cables with losses up to $43 \mathrm{~dB}$.

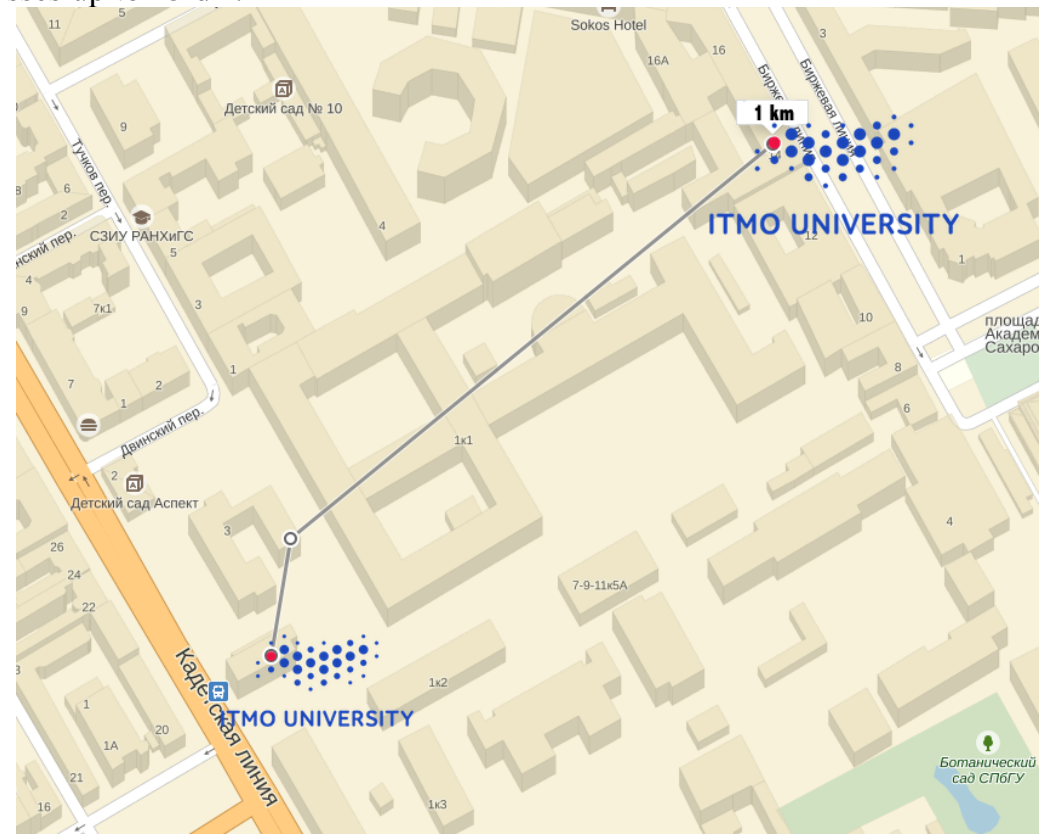

Figure 1. Map showing the network node locations in Saint Petersburg

Notable, these results were achieved using a relatively low clock frequency $100 \mathrm{MHz}$ (an order of magnitude lower than in alternative solutions). It significantly simplifies the electronic subsystem and in the future will allow increasing transmission parameters 3-5 times.

Future work will focus on increasing the number of nodes in order to form a quantum network in metropolitan telecommunication infrastructure.

\section{References}

1. C. Bennett, G. Brassard Proceedings of IEEE International Conference on Computers, Systems and Signal Processing, P. 175 (1984).

2. M. Peev, C. Pacher, R. Alléaume et al. New Journal of Physics 11, 075001, (2009)

3. http://www.toshiba-europe.com/research/crl/qig/quantumkeyserver.html

4. A.V. Gleim, A.A. Anisimov, L.N. Asnis, Yu.B. Vaktkhtomin, A.V. Divochii, V.I. Egorov, V.V. Kovaluk, A.A. Korneev, S.M. Kynev, Yu.V. Nazarov, R.V. Ozhegov, A.V. Rupasov, K.V. Smirnov, M.A. Smirnov, G.N. Gol'tsman, S.A. Kozlov Bulletin of the Russian Academy of Sciences. Physics, 78, № 3,171-175 (2014).

5. J.-M. Mérolla, Y. Mazurenko, J.-P. Goedgebuer, H. Porte, and W. T. Rhodes, Opt. Lett. 24, 104106 (1999)

6. José Mora, Antonio Ruiz-Alba, Waldimar Amaya, Alfonso Martínez, Víctor García-Muñoz, David Calvo, and José Capmany Optics Letters, 37, № 12, (2012) 